\title{
Impact of Induction Chemotherapy in Locally Advanced HPV-negative Oropharyngeal Cancer. A Propensity Score-matched analysis
}

\author{
FRANCESCA DE FELICE ${ }^{1}$, GESSICA ABATE ${ }^{1}$, ALESSANDRO GALDIERI ${ }^{1}$, \\ NADIA BULZONETTI ${ }^{1}$, DANIELA MUSIO ${ }^{1}$ and VINCENZO TOMBOLINI ${ }^{1,2}$ \\ ${ }^{1}$ Department of Radiotherapy, Policlinico Umberto I, Sapienza University of Rome, Rome, Italy; \\ ${ }^{2}$ Spencer-Lorillard Foundation, Rome, Italy
}

\begin{abstract}
Aim: To estimate the clinical outcomes of induction chemotherapy (IC) followed by standard chemoradiotherapy (CRT) and CRT alone in patients with locally advanced human papilloma virus (HPV)-negative oropharyngeal squamous cell carcinoma. Patients and Methods: Consecutive patients with histologically-proven $H P V$-negative squamous cell carcinoma of the oropharynx were included and treated with IC-CRT or CRT alone. In order to compare treatment outcomes and toxicity between groups, patients were matched by primary tumor site and clinical disease stage. Overall survival (OS), disease-free survival (DFS) and metastasis-free survival (MFS) curves were calculated with the Kaplan-Meier method. Results: Nine IC patients and 18 CRT patients were included. All patients completed the programmed treatment. The median follow-up was 38 months. There were no statistically significant differences in OS and DFS between groups. The 5-year MFS was $88.9 \%$ and $50.8 \%$ in the IC-CRT group, respectively. There was no meaningful difference in toxicity between patients. Conclusion: In HPV-negative patients with locally advanced oropharyngeal cancer, adding IC to standard CRT may increase the MFS rate. However no significant differences in OS and DFS were observed. More studies are needed to better elucidate the role of IC in this setting.
\end{abstract}

Despite changes in epidemiology from smoking and alcohol history to human papilloma virus (HPV)-associated disease, oropharyngeal cancer remains one of the most common

Correspondence to: Francesca De Felice, Department of Radiotherapy, Policlinico Umberto I, "Sapienza" University of Rome, Viale Regina Elena 326, 00161 Rome, Italy. Tel: +39 0649973411, Fax: +39 0649973411, e-mail: fradefelice@hotmail.it

Key Words: Induction chemotherapy, locally advanced, head and neck cancer, HPV, oropharyngeal cancer, metastasis, survival, outcomes, chemoradiotherapy, radiation. squamous cell carcinoma, of the head and neck district (1). Concomitant platinum-based chemoradiotherapy (CRT) is still considered the standard treatment approach, due to its organpreservation intent (2). However, scientific research is extremely active in the optimization of the best strategy for a favorable balance between survival and morbidity, primarily for patients with locally advanced disease. Induction chemotherapy (IC) is an area of ongoing interest but also of controversy. There are no evidence-based data suggesting that IC is superior in terms of clinical outcomes to CRT alone and the major criticism is whether IC could negatively impact on the subsequent optimal delivery of planned CRT (3). However, given the superiority of IC using taxane, cisplatin and fluorouracil (TPF) over cisplatin/fluorouracil-based IC, several trials have directly compared TPF-IC followed by CRT with concomitant CRT (4-8). Globally, IC was not associated with better survival rates, but the vast majority of trials included patients treated with TPF followed by non-standard concurrent CRT regimens (4-7). Therefore, the true value of IC remains an unresolved clinical issue. Moreover, there is increasing interest in HPV status. HPV etiology (HPV-positive) appears to be associated with better clinical outcomes, suggesting that intensification of treatment strategy may be necessary in patients with HPV-negative squamous cell carcinoma (9).

Thus, the purpose of this study was to describe the first propensity score-matching analysis of patients with HPVnegative locally advanced oropharyngeal cancer treated with IC followed by standard CRT and those treated with CRT alone at a single institute. The focus was to characterize the influence of IC on the risk of distant metastasis, and in order to produce more robust results, we analyzed metastasis-free survival (MFS) as a surrogate endpoint for overall survival (OS).

\section{Patients and Methods}

Patient selection. This study was approved by the Department of Radiological Sciences, Oncology and Pathology, Policlinico Umberto I, Sapienza University of Rome (prot.443/16) and informed consent was 
waived. Consecutive patients with histologically proven HPV-negative squamous cell carcinoma of the oropharynx were included, provided they were $\geq 18$ years of age, had Eastern Cooperative Oncology Group performance status $\leq 1$, had adequate renal, hepatic and bone marrow function, had locally advanced disease, without any evidence of distant metastases by imaging modalities. Patients were excluded from the study in cases of synchronous tumors, severe comorbidities (cardiovascular disease, history of neurological or psychiatric disorders, collagenosis) or previous radiotherapy to the head and neck region.

Clinical examinations including nasopharyngolaryngoscopy were combined with radiological imaging to assess the precise local (T), regional nodal $(\mathrm{N})$, and distant $(\mathrm{M})$ extent of the tumor. Patient tumors were classified according to the American Joint Committee on Cancer tumor, nodes, metastasis (TNM) Staging System (10). Both preventive dental care and nutrition evaluations occurred before treatment.

Treatment plan. All patients received CRT with curative intent with or without prior IC administration.

IC consisted of three cycles of 21 days each of cisplatin $\left(75 \mathrm{mg} / \mathrm{m}^{2}\right.$, day 1), docetaxel $\left(75 \mathrm{mg} / \mathrm{m}^{2}\right.$, day 1) and 5-fluorouracil $\left(750 \mathrm{mg} / \mathrm{m}^{2} /\right.$ day, days 1-5). Patients were treated with CRT within 3 to 7 weeks after the end of the third IC cycle.

CRT consisted of a standard regimen with $100 \mathrm{mg} / \mathrm{m}^{2}$ cisplatin (days 1, 22 and 43) plus radiotherapy (2 Gy once daily fraction) at a total dose of $70 \mathrm{~Gy}$ to macroscopic disease plus high-risk regions and $50 \mathrm{~Gy}$ to sites of potential disease. Radiation therapy was delivered with intensity modulated (IMRT) technique with 6-15 MV energy photons.

Follow-up. Clinical assessment was carried out every 3 weeks during the IC period, whereas during CRT, patients were evaluated daily. Toxicity was evaluated using National Cancer Institute's Common Terminology Criteria for Adverse Events version 4.0 (11). After treatment, all patients were monitored at three-month intervals for the first 2 years and at 6-month intervals for the subsequent years in order to evaluate toxicities and detect locoregional or distant recurrent disease.

Propensity score-matching analysis. To perform a comparative analysis, patients were selected from our institutional head and neck unit database. Since patients were not randomly assigned to receive IC followed by CRT (IC-CRT group) or CRT alone (CRT group), a propensity score-matching analysis was used to reduce the effect of treatment selection bias and simulate the effects of randomization. A 1:2 matching of IC-CRT to CRT patients was performed to improve statistical power. CRT patients were matched with IC-CRT patients based on several factors, including primary tumor site (tonsil versus base of tongue), $\mathrm{T}$ stage (T1-2 versus $\mathrm{T} 3-4)$ and $\mathrm{N}$ stage (N0-1 versus $\mathrm{N} 2-3$ ).

Statistical analysis. Statistical analysis was performed using the RStudio-0.98.1091 software. Standard descriptive statistics were used to evaluate the distribution of each potential factor. Continuous data are given as the median (range), and categorical data as the number of observations and ratios. Data were compared using nonparametric Fisher exact test for qualitative data and Student $t$-test for quantitative data. OS, disease-free survival (DFS) and metastasis-free survival (MFS) were calculated in months from the date of the end of CRT to the first event, including date of the last follow-up or death (OS), relapse (DFS), or metastasis (MFS). OS,
Table I. Baseline characteristics of the patient population.

\begin{tabular}{lrrrr}
\hline & \multicolumn{3}{c}{ Patients (\%) } & \\
\cline { 2 - 4 } Characteristics & All & IC-CRT & CRT & $p$-Value \\
\hline Median age (range), years & $63(41-83)$ & $65(54-74)$ & $59(41-83)$ & \\
Gender & & & & 0.84 \\
$\quad$ Male & $19(70.4)$ & $6(66.7)$ & $13(72.2)$ & \\
$\quad$ Female & $8(29.6)$ & $3(33.3)$ & $5(27.8)$ & \\
Primary tumor site & & & & 0.50 \\
$\quad$ Tonsil & $21(77.8)$ & $8(88.9)$ & $13(77.2)$ & \\
$\quad$ Base of tongue & $6(22.2)$ & $1(11.1)$ & $5(27.8)$ & \\
Clinical tumor stage (T) & & & & 0.31 \\
$\quad$ T1-2 & $9(33.3)$ & $4(44.4)$ & $5(27.8)$ & \\
$\quad$ T3-4 & $18(66.7)$ & $5(55.6)$ & $13(77.2)$ & \\
Clinical nodal stage (N) & & & & 0.81 \\
$\quad$ N0-1 & $6(22.2)$ & $2(22.2)$ & $4(22.2)$ & \\
$\quad$ N2-3 & $21(77.8)$ & $7(77.8)$ & $14(77.8)$ & \\
\hline
\end{tabular}

IC: Induction chemotherapy; CRT: chemoradiotherapy.

DFS and MFS were estimated using the Kaplan-Meier method and survival curves were compared using the log-rank test (12). All reported $p$-values are two-sided, and $p$-values lower than 0.05 were considered significant.

\section{Results}

Patient characteristics. In total, 27 patients who received either IC-CRT $(n=9)$ or CRT alone $(n=18)$ were included in the final analysis. The median age at diagnosis was 63 (range $=41-83$ ) years and 19 patients $(70.4 \%)$ were male. Tumor characteristics were not significantly different between groups, indicating that the matching procedure worked well (Table I).

Treatment compliance. All patients completed the programmed treatment. No patient stopped IC. All patients received the radiation therapy-prescribed total dose. Overall, radiotherapy was interrupted for a mean period of 7 days for acute toxicity in seven patients $(25.9 \% ; n=3$ in the IC-CRT group, $n=4$ in the CRT group). Concomitant chemotherapy for the three patients in the IC-CRT group was suspended indefinitely, and thus they received fewer than three cycles of cisplatin. After the end of CRT, a clinical complete response was observed in 15 patients $(55.6 \%$; $n=4$ in the ICCRT group, $n=11$ in CRT group). Clinical locoregional reevaluation was stable in only one CRT case.

Clinical outcomes. The median follow-up was 38 (range=283) months for the entire cohort, 46 (range=11-83) months for the IC-CRT group and 31 (range=2-72) months for the CRT group. In total, nine patients deaths were recorded, one in the IC-CRT group and eight in the CRT group. Overall, the 5-year 


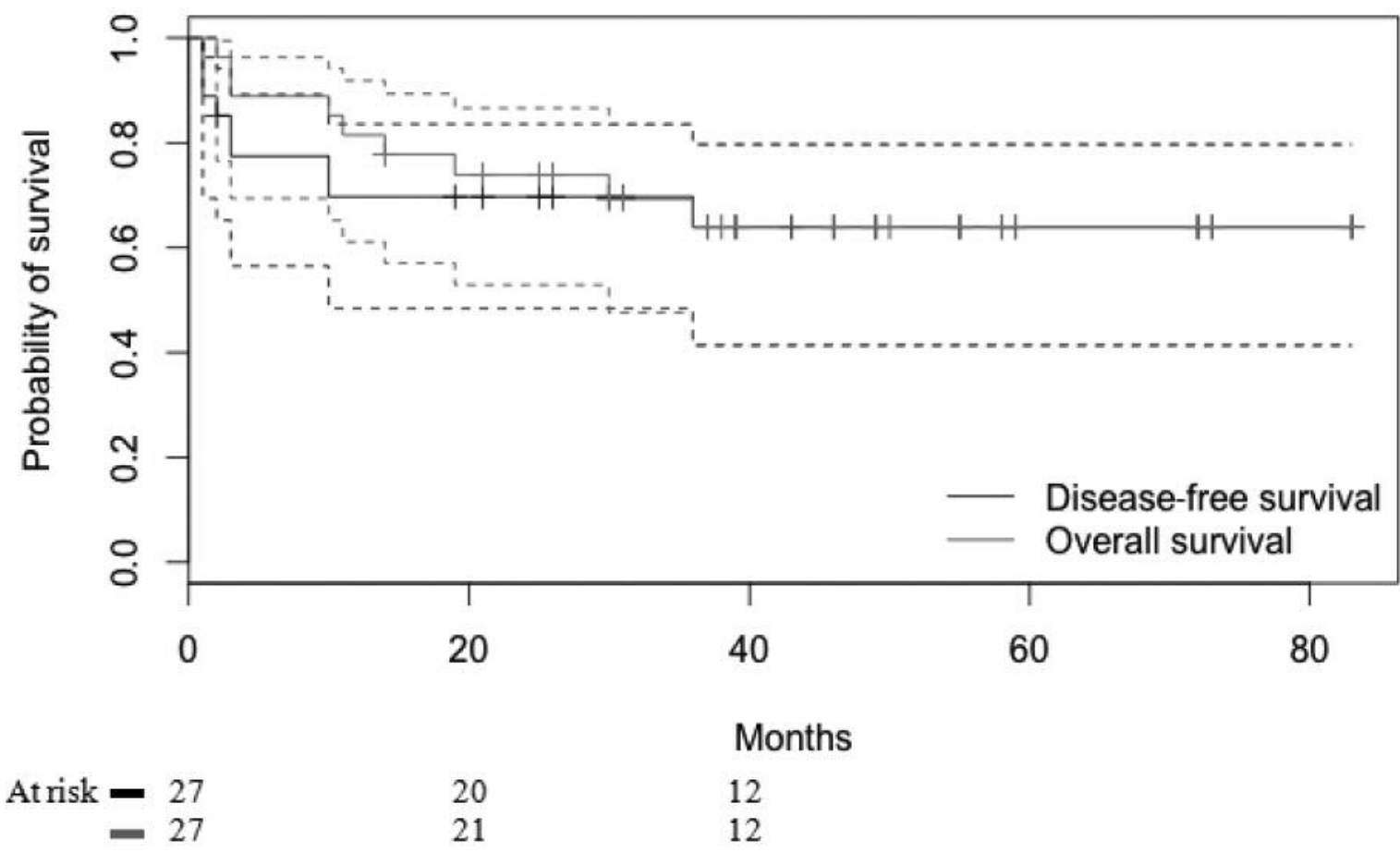

Figure 1. Overall and disease-free survival of the entire cohort.

OS and DFS rates were $63.9 \%$ [95\% confidence interval $\mathrm{CI})=42-80 \%]$ and $63.9 \%(95 \% \mathrm{CI}=41-80 \%)$, respectively (Figure 1). No significant difference were recorded between the IC-CRT and CRT groups in term of OS (88.9\% vs. $50.4 \%$, $p=0.08)$ and DFS $(88.9 \%$ versus $49.6 \%, p=0.09)$.

Distant metastasis occurred in six patients, one in the ICCRT group and five in the CRT group, resulting in a 5-year MFS rate of $88.9 \%$ (95\% CI=43-98\%) in the IC group and $50.8 \%(95 \% \mathrm{CI}=24-72 \%)$ in the CRT group $(p=0.09)$. MFS curves based on treatment group are shown in Figure 2.

Toxicity. All patients had acute toxicity associated with treatment. Details are shown in Table II.

The incidence and severity of oral mucositis seemed to be improved in the IC-CRT group compared to standard CRT. Overall, the significant differences in severe toxicity between the two groups were for grade 3-4 radiation dermatitis $(p=0.05)$ and oral pain $(p=0.02)$. The most important late local toxicities among surviving patients in both groups were xerostomia and dysgeusia.

\section{Discussion}

This case-matched analysis of patients with HPV-negative oropharyngeal squamous cell carcinoma treated with or without IC prior to standard CRT suggests that IC may achieve improvement in distant disease control, while preserving similar toxicity rates. We found that patients who had IC followed by platinum-based CRT had lower rates of distant metastases than those who had received CRT alone. Although the differences were non significant, the OS and DFS rates for the IC-CRT group were also higher than those for the CRT group. With regard to toxicity, there was no meaningful difference in toxicity between patients who had IC and those who did not. Severe oral mucositis and oral pain were the major acute adverse events associated with IC.

These results suggest that IC could be of benefit to selected patients. Based on the consolidate assumption that clinical trials should be designed specifically for tumor HPV status, and in order to achieve the highest quality of research and to generate valid hypothesis, we considered only HPVnegative patients in this analysis (13). The advent of IC has heralded a new era in the treatment paradigm for locally advanced oropharyngeal cancer. Although a poor prognosis of patients with HPV-negative carcinoma has been detected, it remains unclear if patients would benefit from IC. No direct evidence from clinical randomized trials a currently exists to compare IC plus CRT to concurrent standard CRT for HPV-negative patients.

To our knowledge, this is the first study that tested the clinical benefit associated with the use of standard chemotherapy regimen -in both induction and concomitant 


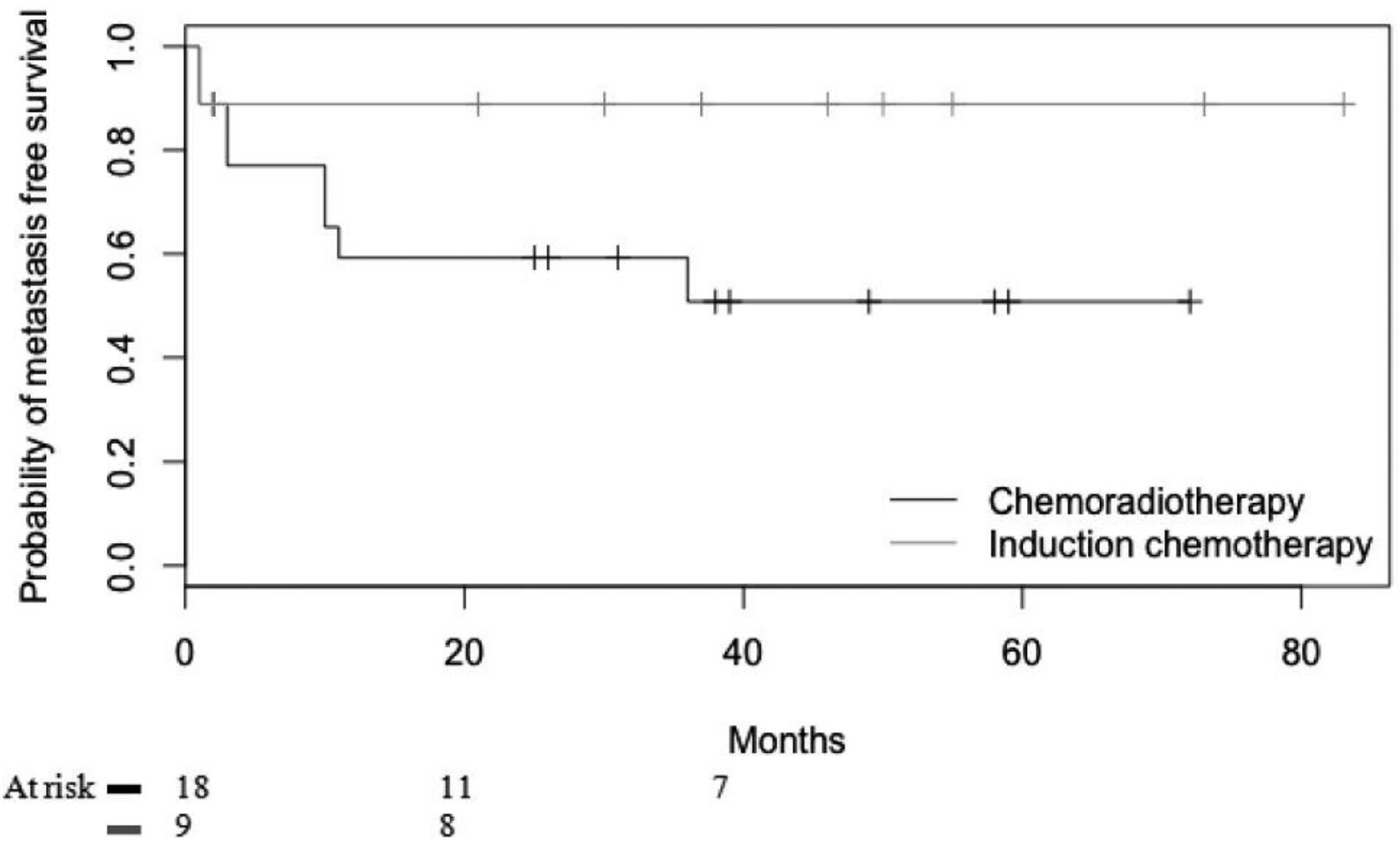

Figure 2. Metastasis-free survival according to treatment group.

Table II. Acute and late toxicity after therapy.

\begin{tabular}{|c|c|c|c|c|c|c|}
\hline \multirow[b]{2}{*}{ Toxicity } & \multicolumn{3}{|c|}{ Grade $1-2$} & \multicolumn{3}{|c|}{ Grade 3-4 } \\
\hline & IC-CRT, n (\%) & CRT, n (\%) & $p$-Value & IC-CRT, n (\%) & CRT, n (\%) & $p$-Value \\
\hline \multicolumn{7}{|l|}{ Acute } \\
\hline Radiation dermatitis & $3(33.3)$ & $8(44.4)$ & 0.55 & $2(22.2)$ & $0(0)$ & 0.05 \\
\hline Mucositis oral & $3(33.3)$ & $9(50)$ & 0.56 & $6(66.7)$ & $9(50)$ & 0.56 \\
\hline Odinophagia/oral pain & $3(33.3)$ & $15(83.3)$ & 0.01 & $6(66.7)$ & $3(16.7)$ & 0.02 \\
\hline Xerostomia & $8(88.9)$ & $13(72.2)$ & 0.27 & $0(0)$ & $0(0)$ & - \\
\hline Dysgeusia & $8(88.9)$ & $15(83.3)$ & 0.79 & $0(0)$ & $0(0)$ & - \\
\hline Dysphagia & $6(66.7)$ & $8(44.4)$ & 0.18 & $2(22.2)$ & $6(33.3)$ & 0.52 \\
\hline Neutrophil count decrease & $1(11.1)$ & $1(5.6)$ & 1.0 & $0(0)$ & $1(5.6)$ & 0.67 \\
\hline \multicolumn{7}{|l|}{ Late } \\
\hline Xerostomia & $7(77.8)$ & $11(61.1)$ & 0.54 & $0(0)$ & $0(0)$ & - \\
\hline Dysgeusia & $1(11.1)$ & $7(38.9)$ & 0.14 & $0(0)$ & $0(0)$ & - \\
\hline Dysphagia & $3(33.3)$ & $4(22.2)$ & 0.51 & $0(0)$ & $0(0)$ & - \\
\hline Peripheral neuropathy & $0(0)$ & $4(22.2)$ & 0.18 & $0(0)$ & $0(0)$ & - \\
\hline Trismus & $0(0)$ & $1(5.6)$ & 0.67 & $0(0)$ & $0(0)$ & - \\
\hline Osteoradionecrosis & $0(0)$ & $1(5.6)$ & 0.67 & $0(0)$ & $0(0)$ & - \\
\hline
\end{tabular}

IC: Induction chemotherapy; CRT: chemoradiotherapy.

phases- and radiation fractionation in this setting of patients. It was highly likely that the two patients groups would have significant baseline differences that could confound the analysis of final outcomes. In order to reduce the effect of treatment selection bias and simulate the effects of randomization, we performed a propensity score analysis. The considerable delay in distant metastasis in the IC-CRT group was consistent with meta-analysis of Chemotherapy in 
Head and Neck Cancer results (MACH-NC) (HR=0.73, $p=0.001$ ) (14). These findings suggest mechanisms for potential synergy among IC and CRT to influence the incidence of distant metastasis. We hypothesized that reinforcement of systemic control with standard recommended treatment could be an additional strategy to improve survival of patients with HPV-negative locally advanced oropharyngeal cancer. A recent meta-analysis addressed the question of whether IC followed by concurrent CRT is superior to in term of clinical outcomes to definitive CRT in head and neck cancer (15). The lack of a significant survival benefit indicates we should stop the administration of induction TPF in clinical practice. But there are several limitations to this meta-analysis and thus one must be cautious when interpreting the results. Although the heterogeneity was $0 \%$, it should be noted that different concurrent chemotherapy schemes and a non-standard CRT regimen were used $(4,5)$. Thus it is debatable whether the non-standard CRT schedule could contribute to reducing the absolute survival difference. However, the potential oncological advantages of IC should not be neglected due to fear of an increase in toxicity rates or reduction in treatment compliance. In fact, in our analysis, the compliance, as well as treatment-related side-effects were comparable in the two groups of patients, indicating that the technical feasibility and safety of IC followed by CRT is similar to those of concomitant CRT alone. Takacsi-Nagy et al. experienced the same phenomenon in their patients randomized to IC plus CRT or CRT alone (16). Severe toxicity was higher in the IC-CRT group than in CRT patients (40 events versus 30 events, $p=0.008)$, but the authors reported that it was manageable and did not interfere with treatment course.

Notably, our study had several limitations. The analysis was retrospective, even though both patient baseline characteristics and intensity-modulated radiotherapy technique were similar for the two groups. However, HPV data and relatively longer follow-up times represent an essential advantage of the present analysis. That the better survival rate of IC-CRT patients showed only a positive trend may due to the smaller sample size. These data will be potentially useful as a reference in the future to delineate the selective use of IC in the management of HPV-negative oropharyngeal squamous cell carcinoma.

Future research is imperative and a major consideration can be proposed. Clinical strategy requires treatment intensification to improve outcomes, especially in HPVnegative patients. Emphasis should be focused on intensification of the standard treatment approach, which may be more useful than the new investigational systemic therapies but will require further validation. Evaluation of these specific responses may allow future optimization in clinical practice, and, as exploration of IC continues, new recommendations may emerge. A small sample size does limit the conclusions that can be drawn from our study, although the low rates of distant metastasis and toxicity are encouraging. Prospective randomized clinical trials to provide more definitive information regarding IC followed by standard CRT are needed.

Our results revealed that IC followed by standard CRT could affect the MFS in HPV-negative oropharyngeal squamous cell carcinoma. This case-matched analysis could serve as comparison for a new combination therapy being investigated. Further homogeneous studies should be useful in better determining the real value of IC in HPV-negative oropharyngeal cancer.

\section{References}

1 Siegel RL, Miller KD and Jemal A: Cancer statistics, 2016. CA Cancer J Clin 66(1): 7-30, 2016.

2 National Comprehensive Cancer Network Guidelines Head and Neck Cancers Version 1.2016. [http://www.nccn.org/]

3 Blanchard P, Bourhis J, Lacas B, Posner MR, Vermorken JB, Cruz Hernandez JJ, Bourredjem A, Calais G, Paccagnella A, Hitt R, Pignon JP; Meta-Analysis of Chemotherapy in Head and Neck Cancer, Induction Project, Collaborative Group: Taxane-cisplatin-fluorouracil as induction chemotherapy in locally advanced head and neck cancers: an individual patient data meta-analysis of the meta-analysis of chemotherapy in head and neck cancer group. J Clin Oncol 31(23): 2854-2860, 2013.

4 Haddad R, O'Neill A, Rabinowits G, Tishler R, Khuri F, Adkins D, Clark J, Sarlis N, Lorch J, Beitler JJ, Limaye S, Riley S and Posner M: Induction chemotherapy followed by concurrent chemoradiotherapy (sequential chemoradiotherapy) versus concurrent chemoradiotherapy alone in locally advanced head and neck cancer (PARADIGM): a randomised phase 3 trial. Lancet Oncol 14(3): 257-264, 2013.

5 Cohen EE, Karrison TG, Kocherginsky M, Mueller J, Egan R, Huang CH, Brockstein BE, Agulnik MB, Mittal BB, Yunus F, Raez S, Mehra R, Kumar P, Ondrey F, Marchand P, Braegas B, Seiwert TY, Villaflor VM, Haraf DJ and Vokes E: Phase III randomized trial of induction chemotherapy in patients with $\mathrm{N} 2$ or N3 locally advanced head and neck cancer. J Clin Oncol 32: 2735-2743, 2014.

6 Lorch JH, Goloubeva O, Haddad RI, Cullen K, Sarlis N, Tishler R, Tan M, Fasciano J, Sammartino DE, Posner MR; TAX 324 Study Group: Induction chemotherapy with cisplatin and fluorouracil alone or in combination with docetaxel in locally advanced squamous-cell cancer of the head and neck: long-term results of the TAX 324 randomised phase 3 trial. Lancet Oncol 12(2): 153-159, 2011.

7 Geoffrois L, Martin L, Garaud P, De Raucourt D, Miny J, Maingon P, Lafond C, Tuchais C, Sire C, Babin E, Coutte A, Tao Y, Rolland F, Girard-Calais $\mathrm{MH}$ and Bourhis $\mathrm{J}$ : Induction docetaxel platinum 5-FU (TPF) followed by cetuximabradiotherapy (cetux-RT) versus concurrent chemo-radiotherapy $(\mathrm{CT} / \mathrm{RT})$ in patients with $\mathrm{N} 2 \mathrm{~b} / \mathrm{c}-\mathrm{N} 3$ non operated stage III-IV squamous cell cancer of the head and neck (SCCHN): Results of the GORTEC 2007-02 phase III randomized trial. J Clin Oncol 34: (suppl; abstr 6000), 2016. 
8 Hitt R, Grau JJ, López-Pousa A, Berrocal A, García-Girón C, Irigoyen A, Sastre J, Martínez-Trufero J, Brandariz Castelo JA, Verger E, Cruz-Hernández JJ; Spanish Head and Neck Cancer Cooperative Group (TTCC): A randomized phase III trial comparing induction chemotherapy followed by chemoradiotherapy versus chemoradiotherapy alone as treatment of unresectable head and neck cancer. Ann Oncol 25(1): 216-225, 2014.

9 Yom SS, Ganti AK, Dietz A. What's New in Head and Neck Cancer: Key Findings in 2015-2016 From ECCO/ESMO, ASTRO, and the Multidisciplinary Head and Neck Cancer Symposium. Am Soc Clin Oncol Educ Book 35: 176-183, 2016.

10 Edge S, Byrd D, Compton C and Winchester DP (eds.): AJCC Cancer Staging Manual, Seventh Edition. New York, NY: Springer Verlag, 2009.

11 Cancer Therapy Evaluation Program: Common Terminology Criteria for Adverse Events, Version 4.0, 2009. Available from: URL: http://ctep.cancer.gov

12 Schemper M and Smith TL: A note on quantifying follow-up in studies of failure time. Control Clin Trials 17(4): 343-346, 1996.

13 Ang KK, Harris J, Wheeler R, Weber R, Rosenthal DI, NguyenTân PF, Westra WH, Chung CH, Jordan RC, Lu C, Kim H, Axelrod R, Silverman CC, Redmond KP and Gillison ML: Human papillomavirus and survival of patients with oropharyngeal cancer. N Engl J Med 363(1): 24-35, 2010.
14 Pignon JP, le Maître A, Maillard E, Bourhis J; MACH-NC Collaborative Group: Meta-analysis of chemotherapy in head and neck cancer (MACH-NC): an update on 93 randomised trials and 17,346 patients. Radiother Oncol 92(1): 4-14, 2009.

15 Budach W, Bölke E, Kammers K, Gerber PA, Orth K, Gripp S and Matuschek $\mathrm{C}$ : Induction chemotherapy followed by concurrent radio-chemotherapy versus concurrent radiochemotherapy alone as treatment of locally advanced squamous cell carcinoma of the head and neck (HNSCC): A meta-analysis of randomized trials. Radiother Oncol 118(2): 238-243, 2016.

16 Takacsi-Nagy Z, Hitre E, Remenar E, Oberna F, Polgar C, Major $\mathrm{T}$, Godeny M, Fodor J and Kasler M: Docetaxel, cisplatin and 5-fluorouracil induction chemotherapy followed by chemoradiotherapy or chemoradiotherapy alone in stage III-IV unresectable head and neck cancer. Results of a randomized phase II study. Strahlenther Onkol 191(8): 635-641, 2015.

Received September 19, 2016

Revised October 1, 2016

Accepted October 6, 2016 